\title{
Mediation of Exoticin analgesia by a truncated 6-transmembrane of the mu-opioid receptor
}

\author{
Fenfen Qin ${ }^{1}$, Qisheng Wang ${ }^{1}$, Anlong Liu ${ }^{2}$, Yongwei Jiang ${ }^{1}$, Zhonghao Li ${ }^{1}$, Han Zhang ${ }^{1}$, \\ Dengyun Nie ${ }^{1}$, Qingyang Liu ${ }^{1}$, Hui Wang ${ }^{1}$, Shang $\mathrm{Lu}^{1}$, Qian Li ${ }^{1}$, Jian Liu ${ }^{1}$, Shengfeng Lu ${ }^{1}$, \\ and Zhigang $\mathrm{Lu}^{1}$ \\ ${ }^{1}$ Nanjing University of Chinese Medicine \\ ${ }^{2}$ Nanjing University Medical School Affiliated Nanjing Drum Tower Hospital
}

October 29, 2020

\begin{abstract}
Background and Purpose:Mu-opioids remain vastly important for pain treatment, and would represent ideal analgesics if their analgesic effects could be separated from their many side effects.Exoticin, an extract of Murraya exotica, has been shown to have an analgesic effect which can be blocked by naloxone.Here, we hypothesized that the analgesic effect of Exoticin is mediated by 6-transmembrane (6TM) of the mu-opioid receptor. Experimental Approach:We determines the analgesic EC50 of Exoticin by using the hot-plate assay.The analgesia of Exoticin was evaluated after intrathecal administration with mu, delta, and kappa antagonists in C57BL/6 mice. Antinociceptive effects of Exoticin on formalin or Complete Freund's adjuvant (CFA) mediated allodynia and heat-hyperalgesia were assessed in exon 11 mu-opioid knockout (E11 KO) and exon 1/ exon 11 mu-opioid double knockout (E1/E11 KO) mice. Opioid-related side effects, such as respiration depression, constipation, locomotor activity and dependence were assessed.Finally, to verify the effect of $6 \mathrm{TM}$ on Exoticin, the analgesic effect of Exoticin mediated by $6 \mathrm{TM}$ of the mu-opioid receptor was determined by over-expression of the adeno-associated virus (AAV) in E1/E11KO mu-opioid mice. Key Results:Delta, kappa antagonists in WT mice had no impact on Exoticin analgesia, while mice which received the $\mathrm{mu}$ antagonist markedly lowered. Loss of the truncated 6TM variants in the E11 KO mouse eliminated Exoticin analgesia. Restoring expression of a $6 \mathrm{TM}$ variant with an AAV-mMor-1G rescued Exoticin analgesia in E1/E11 KO mouse that no expressed the 7-transmembrane (7TM) variants. Conclusion and Implications:Exoticin analgesia depended upon the expression of exon 11-associated truncated $6 \mathrm{TM}$ variants of mu-opioid receptor.
\end{abstract}

\section{Availability of data}

The data that support the findings of this study are available from the corresponding author upon reasonable request. Some data may not be made available because of privacy or ethical restrictions.

\section{Mediation of Exoticin analgesia by a truncated}

\section{6-transmembrane of the mu-opioid receptor}

Fenfen Qin ${ }^{1,2}$, Qisheng Wang ${ }^{1,2}$, Anlong $\mathrm{Liu}^{3}$, Yongwei Jiang ${ }^{2}$, Zhonghao $\mathrm{Li}^{1,2}$, Han Zhang ${ }^{2}$, Dengyun $\mathrm{Nie}^{1,2}$, Qingyang Liu ${ }^{1,2}$, Hui Wang ${ }^{1,2}$, Shang $\mathrm{Lu}^{1,2}$, Qian $\mathrm{Li}^{2}$, Jian Liu ${ }^{1}$, Shengfeng $\mathrm{Lu}^{2}$, Zhigang $\mathrm{Lu}^{1,2}$

${ }^{1}$ College of Pharmacy, Nanjing University of Chinese Medicine, Nanjing, 210023, Jiangsu, China.

${ }^{2}$ Key Laboratory of Acupuncture and Medicine Research of Ministry of Education, Nanjing University of Chinese Medicine, Nanjing, 210023, Jiangsu, China.

${ }^{3}$ The Center of Diagnosis and Treatment for Joint Disease, Drum Tower Hospital Affiliated to Medical School of Nanjing University, Zhongshan Road 321, Nanjing 210008, Jiangsu, China. 


\section{Correspondence}

Zhigang Lu, College of Pharmacy, Key Laboratory of Acupuncture and Medicine Research of Ministry of Education, Nanjing University of Chinese Medicine, Nanjing, Jiangsu 210023, China.

Email: Luzg@njucm.edu.cn

Fenfen Qin and Qisheng Wang contributed equally to this work.

\section{Abstract}

Backgroundand Purpose: Mu-opioids remain vastly important for pain treatment, and would represent ideal analgesics if their analgesic effects could be separated from their many side effects. Exoticin, an extract of Murraya exotica, has been shown to have an analgesic effect which can be blocked by naloxone. Here, we hypothesized that the analgesic effect of Exoticin is mediated by 6-transmembrane (6TM) of the mu-opioid receptor.

Experimental Approach: We determines the analgesic EC50 of Exoticin by using the hot-plate assay. The analgesia of Exoticin was evaluated after intrathecal administration with mu, delta, and kappa antagonists in C57BL/6 mice. Antinociceptive effects of Exoticin on formalin or Complete Freund's adjuvant (CFA) mediated allodynia and heat-hyperalgesia were assessed in exon 11 mu-opioid knockout (E11 KO) and exon 1/ exon 11 mu-opioid double knockout (E1/E11 KO) mice. Opioid-related side effects, such as respiration depression, constipation, locomotor activity and dependence were assessed. Finally, to verify the effect of $6 \mathrm{TM}$ on Exoticin, the analgesic effect of Exoticin mediated by $6 \mathrm{TM}$ of the mu-opioid receptor was determined by over-expression of the adeno-associated virus (AAV) in E1/E11KO mu-opioid mice.

Key Results: Delta, kappa antagonists in WT mice had no impact on Exoticin analgesia, while mice which received the mu antagonist markedly lowered. Loss of the truncated 6TM variants in the E11 KO mouse eliminated Exoticin analgesia. Restoring expression of a $6 \mathrm{TM}$ variant with an AAV-mMor-1G rescued Exoticin analgesia in E1/E11 KO mouse that no expressed the 7-transmembrane (7TM) variants.

Conclusion and Implications: Exoticin analgesia depended upon the expression of exon 11-associated truncated $6 \mathrm{TM}$ variants of mu-opioid receptor.

Keywords:mu-opioid; 6-transmembrane; Exoticin; analgesia

\section{Summary:}

\section{What is already known:}

opioids are valuable analgesics, but produce respiratory depression and other side effects.

Truncated forms of the mu opioid receptor gene Oprm1 containing only six transmembrane domains (6TM) can mediate a potent analgesia without producing many classical opioid side effects.

The analgesic effects of Exoticin can be blocked by naloxone.

What this study adds:

Exoticin acting at $6 \mathrm{TM}$ of mu opioid receptor can produce antinociception without typical opioid side effects.

\section{Clinical significant:}

Exoticin possess desired analgesic therapeutic effects without typical opioid side effects.

\section{Abbreviations}

6TM, 6-transmembrane; 7TM, 7-transmembrane; 1TM, single transmembrane; s. c., subcutaneous; i.t., intrathecal; CFA, Complete Freund's adjuvant; E11 KO, exon 11 knockout; E1/E11 KO, exon 1/exon 11 knockout; WT, wild type; AAV, adeno-associated virus; MOR, mu opioid receptor; OPRM1, mu opioid receptor gene; IBNtxA, 3-iodobenzoyl-6 $\beta$-naltrexamide; Exoticin; 3,3', $4^{\prime}, 5^{\prime}, 5,6,7,8$-octamethoxyflavone; $\beta$-FNA, 
Beta-Funaltrexamine; NTI, Naltrindole; nor-BNI, nor-Binaltorphimine dihydrochloride; MPE, maximum possible effect; CHO, Chinese hamster ovary; PBS, phosphate-buffered saline; OCT, optimal cutting temperature; TSA, tyramide signal amplification; D3PDH, glyceraldehyde-3-phosphate dehydrogenase; EGFP, enhanced green fluorescent protein; NGS, normal goat serum; RT, room temperature; DAPI, 4',6-diamidino2-phenylindole.

\section{Introduction}

Clinically, opioids are still the first choice for the treatment of moderate to severe pain, the prescription of opioids for chronic pain has increased in recent years[1]. Despite their high efficacy in the context of acute pain, opioids are associated with several troubling side effects, including respiratory depression, reduced gastric motility, and dependence/addiction[2,3]. As a result, one of the main goals of pain research is to identify compounds with similar efficacy but fewer side effects.

There are currently three opioid receptor genes reported, but the vast majority of current opioid analgesics bind and activate the mu opioid receptor (MOR), a 7-transmembrane (7TM) G-protein-coupled receptor[4]. MOR gene (OPRM1) comprises of a wide array of mRNA splice variants[5], these splice variants can be grouped into those coding for full-length 7TM proteins, truncated 6-transmembrane (6TM) proteins, and single transmembrane (1TM) proteins[6-8]. Each of these splice variants is pharmacologically distinct and important. The full-length 7TM variants mediate the analgesic actions of traditional $\mu$ opioids such as morphine and methadone[9]. Although the 1TM variants do not bind opioids, they potentiate opioid action through a chaperone-like action. The role of the truncated $6 \mathrm{TM}$ variants was initially uncovered using 3 '-iodobenzoyl-6 $\beta$-naltrexamide (IBNtxA) that revealed a unique pharmacological profile. Previous studies have shown that the truncated 6TM splice variant mediates IBNtxA analgesia, but unlike morphine doesn't depress respiratory depression or produce physical dependence and shows no reward behavior. It is believed that truncated $6 \mathrm{TM}$ splice variants can mediate analgesia with mechanisms distinct from traditional opioids [10]. The vast majority of current opioid analgesics bind and activate the MOR, a full-length 7TM variants associated with exon 1. Unlike exon 1, exon 11 lacks a transmembrane domain, resulting in a truncated form of the receptor with only 6TM. Disruption of exon 1 of the MOR-1 (exon $1 \mathrm{KO}$ ) eliminates all full-length $7 \mathrm{TM}$ variants and all morphine activity, while the $6 \mathrm{TM}$ variants are still expressed in exon $1 \mathrm{KO}$ mouse, and IBNtxA retains full analgesic activity. Conversely, IBNtxA is inactive in an exon $11 \mathrm{KO}$ mouse lacking the truncated $6 \mathrm{TM}$ splice variants while morphine retains full analgesic activity[9, 11]. The $6 \mathrm{TM}$ variants were an unanticipated drug target, since they do not conform to the traditional structure of GPCRs. It provides a target for drug development, with the possibility of potent analgesics lacking many of the side effect.

Exoticin (3,3', $4^{\prime}, 5^{\prime}, 5,6,7,8$ - octamethoxyflavone), the flavonoids extract of Murraya exotica, is widely distributed in India, Southeast Asia, and Southern China[12]. The leaves and roots of theMurrayaplants have been used as folk medicines for the treatment of analgesia, anesthesia, abdominal pain, rheumatism, etc. Pharmacological studies showed the antioxidant, antimicrobial, antitumor and anti-inflammatory activities for the extracts from Murraya exotica [13-15].Flavonoids have been reported to be the main bioactive constituents in Murraya exotica, along with several Coumarins and alkaloids[16-18]. Previous studies have shown that Exoticin acting on opioid receptors[19], the current study focuses on if Exoticin analgesia mediated by $6 \mathrm{TM}$ alternative splice variants of the $\mu$ opioid gene.

\section{Experimental Procedures}

2.1 Animals. Exon $11 \mathrm{KO}$ (E11 KO) mice with disruptions of exons 11, Exon 1/exon $11 \mathrm{KO}$ (E1/E11 KO) mice with disruptions of exons 1 and exons 11, both were a gift from Ying-Xian Pan, Memorial SloanKettering Cancer Center, New York, NY, USA. C57BL/6 mice were obtained from the Experimental Animal Center of Nanjing University of Chinese Medicine, Nanjing, China. Animals were housed in a temperaturecontrolled environment on a 12h light/dark cycle with access to food and water and a standard laboratory diet. All animals' treatment protocols were seriously following the International ethical guidelines and the National Institute of Health Guide concerning the Care and Use of Laboratory. All experiment was approved by the committee on the Ethics of Laboratory Animal Experiments of Nanjing University of Chinese Medicine 
(NO. 202008A022).

2.2 Drugs. Exoticin was purchased from Shanghai yuan ye Bio-Technology Co., Ltd (CAS NO.13364-94-8, Shanghai, China). Naloxone was purchased from Jiangsu Provine Hospital of Chinese Medicine (Nanjing, China). Beta-Funaltrexamine ( $\beta$-FNA, irreversible and selective $\mu$ antagonist), Naltrindole hydrochloride (NTI, a highly potent and selective non-peptide $\delta$ opioid antagonist) and nor-Binaltorphimine dihydrochloride (nor-BNI, selective $x$ antagonist) were purchased from Tocris Bioscience. Morphine was obtained from Jiangsu Medicine Co., LTD. $\beta$-FNA (40 mg/kg, s. c) and nor-BNI $(10 \mathrm{mg} / \mathrm{kg}$, s. c) were administered $24 \mathrm{~h}$ before agonist testing. NTI $(0.5 \mathrm{mg} / \mathrm{kg}, \mathrm{s} . \mathrm{c})$, naloxone $(1 \mathrm{mg} / \mathrm{kg}, \mathrm{s} . \mathrm{c})$, were administered $15 \mathrm{~min}$ before Exoticin.

2.3 Adenovirus associated virus (AAV) and Administration.Adenovirus associated virus was obtained from Shanghai Genechem Co., Ltd. The AAV was generated by using constructs containing EGFP with and without the mMOR-1G. It was given by intrathecal (i.t.) injections that were performed by lumbar puncture using a $10 \mu \mathrm{L}$ syringe under general halothane anesthesia. The $2 \mu \mathrm{L}$ of AAV expressing mMOR-1G or vector alone without insertion $(1.83 \mathrm{E}+13 \mathrm{v} . \mathrm{g} . / \mathrm{ml})$ were administrated intrathecally in each mouse on days 1,3 , and 5 , as previously described[10].

\subsection{Nociceptiveassays}

2.4.1 Tail-Flick Assays. Opioid analgesia was performed with a heat tail-flick assay, with a maximal latency of $10 \mathrm{sec}$ to prevent tissue damage, Baseline latencies typically ranged around 3-4 sec. And data are presented as percent maximum possible effect (\%MPE) according to the formula: \% MPE $=[($ latency after drug - baseline latency $) /(10$ - baseline latency $) * 100]$. Experiments were performed at least three times with similar results observed during each replication unless stated otherwise[20, 21]. Exoticin was administered subcutaneously (s. c.) at the indicated doses and analgesia testing carried out 30 min later at peak effect. To examine antagonism, the drug (s. c.) was given $15 \mathrm{~min}$ before Exoticin $(32 \mathrm{mg} / \mathrm{kg}$, s. c.) and analgesia assessed $30 \mathrm{~min}$ after Exoticin.

2.4.2 Hot-Plate Test. The temperature of the hot-plate was set at $54 \pm 1{ }^{\circ} \mathrm{C}$. Mice were placed inside a transparent Plexiglas cylinder on a metal surface to observe its pain responses (hind-paw-licking or jumping). The latency time to respond shorter than $5 \mathrm{~s}$ or longer than the 30s were excluded from the test. This test was performed only once, as repeated testing leads to systematic latency alterations, as previously described. Exoticin doses used in cumulative dose-response studies were $8,16,32,48$, or $64 \mathrm{mg} / \mathrm{kg}$. EC50 values with 95\% confidence intervals were determined using nonlinear regression analysis (GraphPad Prism8).

2.4.3 Von Frey test. In a quiet environment, the mice were placed in a special box with a mesh bottom. After 15 min of adaptation, a von Frey filament was used to stimulate the middle part of the mouse's paw under the skin to induce a reaction and until the hind paw was withdraw. The minimal value that caused at least three responses was recorded as the mechanical pain threshold. The interval between each application was $5 \mathrm{~min}$. Mice were tested for baseline sensitivity, injected with Exoticin, and then retested at 0.5, 1, 2, 4, and 6 hours after injection. At each time point, 3 withdrawal determinations for each hind paw were made and averaged.

2.4.4 Formalin Test. Mice were habituated for at least 30 minutes to individual cylinders. Mice were then removed from their cylinder, injected with Exoticin, and placed back in the cylinder. Fifteen minutes later, all subjects were given a subcutaneous injection of $5 \%$ formalin (20 $\mu \mathrm{L}$ volume) into the plantar right hind paw and recorded for 60 minutes. Immediately after the formalin injection, the mice were placed individually in the cylinders, and a mirror was placed under the cylinders to allow clear observation of the paws of the animals. The time that the animals spent on biting/licking in the injected paw were measured with a stopwatch every $5 \mathrm{~min}$. The early phase of the formalin test was defined as 0 to 10 minutes after injection, and the late phase as 10 to 60 minutes after injection. Data are presented with the time of samples in each phase in which licking or biting was detected.

2.4.5 Complete Freund's adjuvant-induced mechanical allodynia.Mice were injected with complete 


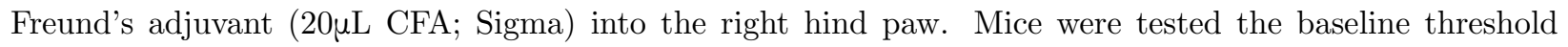
of hotplate and mechanical on day 4 after CFA injection. Immediately thereafter, they were injected with Exoticin, returned to their cubicles, and retested on the hot-plate and mechanical test at $0.5,1,2,4$, and 6 hours after injection.

\subsection{Opioid-related side effects}

2.5.1 Dependence. Mice were injected daily with Exoticin $(32 \mathrm{mg} / \mathrm{kg}$, s. c.) or Morphine (10 mg/kg s. c.). On day 7 of chronic daily Exoticin dosing, mice were injected with naloxone (1 mg/kg, s. c.) and jumping was quantified for the next 15 minutes[22, 23].

2.5.2 Respiratory Depression. Respiratory rate was assessed in freely moving adult mice with the Wholebody plethysmography (WBP) system (Buxco@ Fine-Pointe, USA). The integrated software analysis was used for calculation of the respiratory frequency and tidal volumes. Mice were habituated to the device for at least 15 min before testing. A baseline was obtained over 10 minutes before drug injection. After baseline measurement, mice were injected with vehicle, Exoticin $(32 \mathrm{mg} / \mathrm{kg}$, s. c.), or morphine (5 mg/kg, s. c.) and were put back into the recording chamber to measure respiration parameters. Testing began 10 minutes post-injection. These parameters were frequency of breathing (f), minute tidal volume (MVb).

2.5.3Fecal accumulation experiment. Mice were provided with food and water ad libitum prior to the test. Mice were treated with Exoticin $(32 \mathrm{mg} / \mathrm{kg}$, s. c.) or morphine $(5 \mathrm{mg} / \mathrm{kg}$, s. c.) and then individually placed in a Plexiglas box with a wired mesh or grid floor. Fecal were collected in a metal tray and weighed at 1-h intervals.

2.5.4 Locomotor activity assay. Locomotor activity was assessed using open field activity chambers. Animals were habituated to the testing room for at least 1 hour prior to testing. On Day 1, animals were injected with saline and immediately placed in the activity chamber; at the same time on Day 2, the same animals received Exoticin (32 mg/kg, s.c.) or morphine (5 mg/kg, s.c.). Immediately, their movements were recorded for 1 hour following injection using VisuTrack software.

\subsection{Binding assays}

2.6.1 Receptor binding.Membrane receptor proteins are prepared by membrane extracted from Chinese hamster ovary cells (CHO cells). Add about 50ug of membrane receptor protein and $\left[{ }^{3} \mathrm{H}\right]$-labeled ligand $(1$ $-2 \mathrm{nM})$ to the total binding tube, add $1 \mu \mathrm{M}$ of the corresponding ligand to the corresponding non-specific binding tube, and add different concentrations to the sample tube. A screened opioid ligand drug with a final volume of $100 \mu \mathrm{l}$, incubate at $30^{\circ} \mathrm{C}$ for $30 \mathrm{~min}$, and stop the reaction in ice water. Filter on Millipore sample collector through GF/C (whatman) glass fiber filter paper under vacuum. Rinse three times with $4 \mathrm{ml} 50 \mathrm{mM}$ Tris- $\mathrm{HCl}(\mathrm{pH} 7.4)$, dry the filter paper, place it in a $0.5 \mathrm{ml}$ Eppendorf tube, add 0.5ml lipophilic scintillation fluid, PERKIN ELMER PRI-CARB 2910 liquid scintillation counter to measure the radioactivity and calculate the inhibition rate, the experiment was repeated more than three times, with three duplicate tubes in each group. IC50 values were determined and converted to Ki values. Specific binding was defined as the difference between total binding and nonspecific binding, Calculate according to the following formula:

Inhibition rate $=($ total binding tube dpm-sample tube $\mathrm{dpm}) /($ total binding tube dpm-non-specific binding tube-non-specific binding tube dpm) $\times 100 \%$

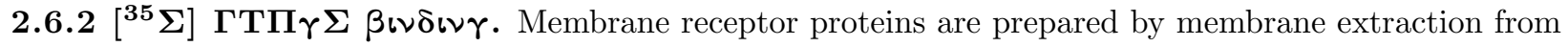
$\mathrm{CHO}$ cells. Protein concentrations were determined using the BCA (Bicinchonininc acid) method. The prepared membrane receptor protein is diluted to the desired concentration with reaction buffer. Incubate the reaction tube in $27^{\circ} \mathrm{C}$ water bath for 1 hour, filter under reduced pressure with a glass fiber membrane and perform liquid scintillation counting. The basal level of binding was determined by incubation of samples in the absence of agonists. Non-specific binding was determined in the presence of $100 \mathrm{mM}$ un-labelled GTP?S.

Calculate according to the following formula:

$\left[{ }^{35} \mathrm{~S}\right]$ GTP?S binding rate $=100 \times\left(\mathrm{cpm}_{\text {sample}}-\mathrm{cpm}_{\text {non-specific }}\right) /\left(\mathrm{cpm}_{\text {basal }}-\mathrm{cpm}_{\text {non-specific }}\right)$ 
2.7 Reverse transcription and polymerase chain reaction (RT-PCR). Total RNA was extracted from the spinal cord using TRIzol reagent following the protocols supplied by the manufacturer. Brief, after deep anesthesia was induced using pentobarbital $(100 \mathrm{mg} / \mathrm{kg}$, i. p.), the mouse L3-L5 spinal cord was collected and homogenized in $1 \mathrm{ml}$ of Trizol (Takara, Japan) on ice. Total RNA $(1 \mu \mathrm{g})$ was reverse-transcribed into cDNA using PrimeScript RT kit (Toyobo, Japan). Then, real-time PCR was conducted using SYBR@ Green (Takara, Japan) following the manufacturer's instructions. The following primer sets were used: the forward primer of 5'-GGA CGG CAA CAT CCT GGG GC-3' and reverse primer of 5'-CGT TGG GGT CTT TGC TCA GGG C-3' for EGFP; the forward primer of 5'-ACC ACA GTC CAT GCC ATC AC-3' and reverse primer of 5'-TCC ACC ACC CTG TTG CTG TA-3'for G3PDH. PCR products were separated on $2 \%$ agarose gel containing $66 \mathrm{pg} / \mathrm{ml}$ ethidium bromide and imaged using Tanon system (Tanon). To quantify the expression of EGFP mRNA, we used SYBR green quantitative PCR (qPCR) with the same cDNAs and primers as above. The $\mathrm{qPCR}$ program was: initial denaturation at $95^{\circ} \mathrm{C}$ for $3 \mathrm{~min}$ then $95^{\circ} \mathrm{C}$ for 15 sec and $60^{\circ} \mathrm{C} 30 \mathrm{sec}$ for 36 cycles. SYBR was used as a fluorescent dye. The threshold cycle, Ct was measured which correlates inversely with the target mRNA level. G3PDH qPCR with the same primers as above was used for normalization. Normalized expression was calculated as $2^{-\Delta \Delta^{\prime \prime}(\tau)}=2^{-\left[\Delta^{\prime \prime}(\tau) Е \Gamma \Phi \Pi-\Delta^{\prime \prime}(\tau) \Gamma 3 \Pi \Delta H\right]}$.

2.8 Immunofluorescence analysis.mice were anesthetized using ketamine ( $150 \mathrm{mg} / \mathrm{kg}$, i. p.) and then perfused intracardially with phosphate-buffered saline (PBS), pH7.4, followed by $4 \%$ paraformaldehyde in PBS, pH7.4. The spinal cords were removed, post-fixed for $2 \mathrm{~h}$ in the same fixative, cryoprotected overnight in $25 \%$ sucrose in PBS, and frozen in Tissue-Tek optimal cutting temperature (OCT). Immunostaining was performed on $15 \mu \mathrm{m}$ frozen section cut on a Leica Cryomicrotome. Briefly, after treatment with $3 \% \mathrm{H}_{2} \mathrm{O}_{2}$ in PBS for $10 \mathrm{~min}$, the section was placed in a blocking solution $(0.1 \mathrm{M}$ Tris $/ \mathrm{HCl}, \mathrm{pH} 7.4$, and $0.15 \mathrm{M} \mathrm{NaCl})$ containing $5 \%$ normal goat serum (NGS) for $1 \mathrm{~h}$ at room temperature (RT). For the double EGFP/beta3Tubulin staining, the section was then incubated with a chicken anti-GFP antibody (1:200, ab13970, Abcam) and a rabbit anti-beta3-Tubulin antibody (1:200, D71G9, Cell Signaling) in blocking solution containing 1\% NGS overnight at $4^{\circ} \mathrm{C}$. The section was incubated with a biotinylated goat-anti-chicken IgG and a biotinylated goat-anti- rabbit IgG (1:500, $2 \mathrm{~h}$ at RT). All sections were cover-slipped with mounting medium containing 4',6-diamidino-2-phenylindole (DAPI).

2.9 Data and statistical analysis. The data and statistical analysis comply with the recommendations of the British Journal of Pharmacology on experimental design and analysis in pharmacology. Unless stated otherwise, all data are presented as mean and error bars indicate the SEM. Statistical significance was set at $\mathrm{P}<0.05$, and analysis was performed using GraphPad Prism 8 software. F tests were used routinely to establish if differences in variance existed between groups. If $\mathrm{F}$ tests showed a significant difference in variance between groups, a nonparametric two-tailed Mann-Whitney U-test was used.

\section{Result}

Analgesic actions of Exoticin. We compiled analgesic dose-response curves for Exoticin using the 54 hot-plate test. Exoticin produced robust and dose-dependent analgesia on the hot-withdrawal test (Figure 1). Exoticin doses of $8,16,32,48$ and $64 \mathrm{mg} / \mathrm{kg}$ were selected for the study. The results showed that $8 \mathrm{mg} / \mathrm{kg}$ of Exoticin did not result in significant anti-hyperalgesic effects on the hot-withdrawal test $(\% \mathrm{MPE}<50 \%$; Figure 1A). However, after $30 \mathrm{~min}$ of subcutaneous injection of $16 \mathrm{mg} / \mathrm{kg}$ Exoticin in WT mice, the withdrawal time of the hot plate was increased, and reached a peak in $1 \mathrm{~h}$, and subsided after $2 \mathrm{~h}$, at this time the MPE value was less than $50 \%$ (Figure 1B). Subcutaneous injection of 32, 48, and $64 \mathrm{mg} / \mathrm{kg}$ Exoticin in WT mice exerts analgesia after $30 \mathrm{~min}$. It reached a peak in $1 \mathrm{~h}$ and subsided after $2 \mathrm{~h}$, but the MPE value was still greater than 50\% (Figure 1C, D, E). After subcutaneous injection of Exoticin, mice can reach the peak of analgesia about $1 \mathrm{~h}$, and the ED50 is $16 \mathrm{mg} / \mathrm{kg}$ (Figure 1G).

Exoticin exerts analgesic actions in inflammatory pain models. We also show analgesic actions for Exoticin on two measures of inflammatory pain, and a reversal of thermal and mechanical allodynia after chronic inflammatory injury. Exoticin produced robust analgesia in the late phase but not the early phase (Figure 2A). In the first phase of pain, WT mice and E1/E11 KO mice showed similar abnormal behavior after formalin injection, and there was no significant difference in abnormal behavior time within 5 minutes, 
and after the Exoticin treatment, the abnormal behavior of pain in WT mice changed obviously, while Exoticin did not have such a good therapeutic effect on the pain of E1/E11 KO mice. For the intermittent period of pain, Exoticin does not effect the behavior of both the mice, neither aggravating nor reducing the performance of pain. During the second phase of pain, we found that Exoticin can significantly reduce the abnormal behavior time of second phase in WT mice. But for E1/E11 mu opioid double KO mice, Exoticin can't play the effect, the total time of abnormal behavior before and after treatment is almost the same.

To see if Exoticin' s efficacy against chronic pain was similarly mediated, we induced thermal and mechanical allodynia in WT and E1/E11KO mice using CFA injections. Both results displayed expected time courses of allodynia, E1/E11 KO mice exhibited significantly higher levels of allodynia after CFA (Figure 2B, C). Finally, despite limited efficacy against chronic pain, Exoticin was fully efficacious in reversing, for at least 120 minutes after injection, fully developed thermal and mechanical allodynia 4 days after CFA injection.

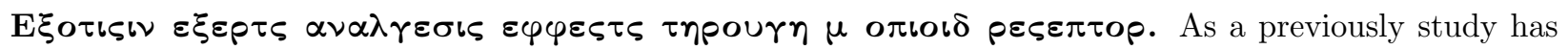
shown, Exoticin exerts its analgesic effect through opioid receptors[19]. Based on the results, we examined the activity of Exoticin in greater detail. To ascertain whether the analgesic effect of Exoticin is through the $\mu$ opioid receptor, we used different antagonists of the opioid receptor. The mu-selective antagonist $\beta$-FNA effectively reversed Exoticin-induced antinociception, whereas the $\delta$ selective antagonist NTI and the $\chi$ antagonist nor-BNI did not. As shown in the Figure 3. Compared with the vehicle group, after intrathecal injection of $\mu$ opioid antagonist ( $\beta$-FNA), the withdrawal time of Exoticin on the hot plate and tail flick of WT mice was significantly reduced, and hot plate MPE only reached about 20\% (Figure 3A), while the withdrawal time for tail flick was almost no difference from baseline (Figure 3B). However, after the use of $\delta$ receptor antagonists (NTI) and $x$ receptor antagonists (nor-BNI), the withdrawal time of Exoticin for hot plate and tail flick on WT mice were not different from that of the Vehicle group (Figure 3A, B). The above results indicate that the analgesic effect of Exoticin is mediated by $\mu$ opioid receptor.

Exoticin analgesia mediated by a truncated 6TM but not 7TM of the MORs. The MOR undergoes extensive splicing, including with the $6 \mathrm{TM}$ and $7 \mathrm{TM}$ variants responsible for a range of analgesia. The current study shown that for thermal allodynia, Exoticin is almost noneffective in E11 KO and E1/E11 KO mice at doses but producing reversal of allodynia in WT mice (Figure 3C, D). Compared with the WT group, the withdrawal time of Exoticin on the hot plate in E11 KO and E1/E11 KO mice were significantly reduced, with MPE\% only reaching about 30\%, while there was almost no difference with baseline in the withdrawal time of tail flick, with MPE\% only reaching about 15\%. That is, after Exoticin administration, the pain threshold of E1/E11 KO mice was significantly reduced, and the analgesic effect of Exoticin was not different from that of the baseline group. The results shown Exoticin is producing pain inhibition across various modalities via $6 \mathrm{TM}$ opioid receptors, indicating that the analgesic effect of Exoticin is mediated by the $6 \mathrm{TM}$ but not 7TM of $\mu$ opioid receptors. Consistent with the notion that Exoticin is producing its analgesic effects on a $6 \mathrm{TM}$ receptor, Exoticin analgesia on the radiant heat tail-withdrawal test was abolished in mice lacking exon 11 of the Oprm1 gene (Figure 3D).

Loss of Exoticin actions in E11 KO mice implicated 6TM variants in its actions. To further verify if a truncated 6TM exon 11 MOR-1 variant could restore Exoticin analgesia in the E1/E $11 \mathrm{KO}$ mice, we observed the recovery of Exoticin's analgesic effect on E1/E11 KO mice by intrathecal injection of AAV. First, we generated an AAV expressing the $6 \mathrm{TM}$ variant mMOR-1G, the predominant $6 \mathrm{TM}$ variant in the spinal cord, along with a co-expressed EGFP marker. An AAV expressing the EGFP marker but lacking the mMOR-1G variant served as a control. Schematic of the virus injection paradigm was shown in Figure 5A, the virus was administered intrathecally on days 1, 3, and 5. Virus expression was seen as early as 2 weeks, it gradually increased over 3 weeks and then remained stable for experimental stage (Figure 5B, C). EGFP labeling was highest in the lumbar/sacral levels, near the site of injection; was colocalized with the marker beta3-Tubulin; and was prominent in the dorsal horns, a region important in pain modulation (Figure 5D).

The expressed mMOR-1G was the most functionally active 4 weeks after AAV injection. Exoticin analgesia returned as early as 2 week following virus infection, gradually increasing until it reached a peak response after 4 weeks (Figure 6A), a time course similar to that of EGFP expression (Figure 5B, C). Mice infected 
with a control virus that did not contain mMOR-1G failed to display an analgesic response (Figure 6B), while Exoticin analgesia returned in the AAV-mMOR-1G infected mice, with a response similar to that seen in WT mice (Figure 6B). Although expression of mMOR-1G rescued Exoticin analgesia, it failed to rescue morphine and fentanyl. Morphine analgesia is independent of exon 11 variants, so this result was anticipated.

Exoticin side effects associated withopioids.Classical opioid analgesics, including morphine, mediate all of their desired and undesired effects by specific activation of the $\mu$ opioid receptor. The use of morphine for treating chronic pain, however, is limited by the development of constipation, respiratory depression, tolerance and dependence. Analgesic effects can also be mediated through other members of the opioid receptor family.

The effect of Exoticin on respiratory function was evaluated by measuring the respiratory rate and tidal volume of WT mice over a period of time. Morphine $(5 \mathrm{mg} / \mathrm{kg} \mathrm{s}$. c.) has a significant inhibitory effect on the respiratory frequency and tidal volume of WT mice, while the Exoticin $(32 \mathrm{mg} / \mathrm{kg} \mathrm{s}$. c.) group of mice has no significant difference compared with the vehicle group, and the results are not statistically significant (Figure 4A, B).

Exoticin's effect on gastrointestinal function was initially assessed by measurement of fecal boli production over time wherein the boli were collected and weighed over a 6 -h period. Morphine $(5 \mathrm{mg} / \mathrm{kg} \mathrm{s}$. c.) induced a suppression of defecation in the WT mice; whereas the WT mice treated with Exoticin continue to produce similar defecation throughout the test period relative to the saline treatment (Figure 4C).

$\mathrm{Mu}$ opioids classically increase locomotion in mice, presumably through the disinhibition of dopamine release in the mesolimbic dopamine pathway[24]. Morphine stimulated locomotion increased is well known. In our studies, Morphine increased locomotion in WT mice (Figure 4D), which begin to rise 10 minutes after injection and lasted for 1 hour. The cumulative distance traveled also did not differ between saline and Exoticin mice in either treatment group. Consistent with the previous view, locomotion was mediated through $7 \mathrm{TM}$ variants and not $6 \mathrm{TM}$ ones.

Lack of physical dependence on Exoticin in WT after short-term repeated administration (Figure 4E). Respectively, Exoticin (32mg/ kg, s. c., daily for 7 days) and Morphine (5 mg/kg s. c., daily for 7 days) were administered once daily for 7 days. On Day 7 , the antagonist naloxone $(1 \mathrm{mg} / \mathrm{kg}$, s. c.) was used to precipitate withdrawal signs that were measured in WT after Exoticin and Morphine treatment. After repeated administration of Exoticin, naloxone did not precipitate withdrawal signs, while morphine showed strong withdrawal symptoms (Figure 4E). That is, Exoticin does not produce physical dependence after 7 days of repeated administration.

Binding data indicates that Exoticin actions independent of 7TM MOR, KOR and DOR and through 6TM. To characterize ligand binding affinities toward different receptors, we performed the Receptor and $\left[{ }^{35} \mathrm{~S}\right] \mathrm{GTP} \gamma \mathrm{S}$ as described in method section. The results are tabulated in Table 1 and Table 2. Exoticin binds to DAMGO, U69593 and DPDPE $(\mathrm{Ki}>1000 \mathrm{nM})$; and GTP $\gamma \mathrm{S}$ binds reveal that Exoticin has no excitability to traditional $\mu$ opioid receptors, and $x$ receptors and $\delta$ receptors $(\mathrm{Ki}>1000 \mathrm{nM})$. This data suggests that Exoticin mediates its actions independent of 7TM MOR, KOR and DOR and through $6 \mathrm{TM}$ unlike IBNtxA which has high affinity and activity at traditional opioid receptors[25, 26].

\section{Discussion and Conclusion}

The relative paucity of novel analgesic compounds displaying clinical efficacy being developed in recent decades has led to much speculation that existing pharmacological mechanisms can't satisfy the research of human clinical treatment of pain drugs. Here we provide evidence that analgesic efficacy of Exoticin, which acts through the $6 \mathrm{TM}$ alternative splice variants of the mouse Oprm1 gene. Dose responses were observed against hot allodynia in WT, meanwhile, acute nociceptive assays, formalin-induced spontaneous pain, hot and mechanical allodynia produced by CFA inflammation injury were performed to ascertain the analgesic effect of Exoticin.

From the behavioral results, we can see that the knockout of $\mu$ opioid receptor genes (E1/E11 KO mice) all 
affected the analgesic effect of Exoticin. From the perspective of the formalin experiment, first-phase pain is a short-lasting but relatively severe pain process caused by formalin[27]. Exoticin can have a significant analgesic effect on formalin-induced pain in WT, but for E1/E11 KO mice, Exoticin does not have an analgesic effect, which can be seen, the knockout of $\mu$-opioid greatly affects the analgesic efficacy of Exoticin. During the intermittent period (5-15min), the mice appear to be relatively calm, so there is no difference in the efficacy and analgesia. For biphasic pain (15-60min), WT mice treated with Exoticin get a better therapeutic effect, while Exoticin almost loses the effect for E1/E11 KO mice. The difference between WT and E1/E11 KO reflects $\mu$-opioid played a decisive role in the analgesic process of Exoticin. We also demonstrate that with $\mu$ opioid receptor blockers, $\beta$-FNA, reduced the withdrawal time of Exoticin on the hot plate and tail-flick in WT mice. However, after the use of $\delta$ and $x$ receptor antagonists, the withdrawal time of Exoticin for hot plate and tail-flick on WT mice was not different from that of the Vehicle group. The above results show that $\mu$-opioids play a decisive role in the analgesic process of Exoticin while also showing that exoticin unlike IBNtxA doesn't label KOR and DOR in vivo[28, 29].

There is a large number of truncated GPCRs that their functions have been unclear[30]. Many have been acted as dominant negatives to modulate the activity of full-length variants, and some have been implicated in disease processes[31]. The 6TM Oprm1 gene variants do not fit into these categories. Rather, they represent a functionally active and previously unrecognized target for opioid analgesics with distinct and improved pharmacology[10, 32, 33]. Classical mu drugs, like morphine and methadone, act only through full-length $7 \mathrm{TM}$ mu variants[5, 11,34]whereas $6 \mathrm{TM}$ variants are both necessary and sufficient for other drugs, as illustrated by IBNtxA[10]. Now, evidence for a nontraditional $\mu$-opioid mechanism of Exoticin analgesia was provided via the demonstration of the analgesic profile of Exoticin in knockout mice (Figure 3C, D). Exoticin analgesia is abolished in mutants lacking exon 11 of the Oprm1 gene. The exon $11 \mathrm{KO}$ mice remain sensitive to morphine and methadone, presumably because of their continued expression of the full-length 7TM variants. The current study reinforces the notion that Exoticin in mice lacking E11-MOR1 variants (E11 KO mice), like IBNtxA, eliminated antinociception, while in mice lacking all mu-opioid receptor splice variants (E1/E11 KO mice), Exoticin antinociception also was eliminated. The results from $\mathrm{KO}$ mice experiments implicate traditional 6TM mu-opioid receptor in Exoticin antinociception.

The current studies describe an exon 1/exon 11 KO mouse with disruptions of both exon 1 and exon 11, yielding a full $\mu$ receptor lacking all Oprm1 splice variants[10] that is insensitive to $\mu$ opioids. Loss of all Oprm1 gene products in the E1/E11 KO mouse eliminated Exoticin analgesia, which could be rescued by the $6 \mathrm{TM} / \mathrm{AAV}$-expressing mMOR-1G, with a rescued response similar to that seen in WT mice. The expressed mMOR-1G was functionally active. Exoticin analgesia returned as early as 2 weeks following AAV infection, gradually increasing until it reached a peak response after 4 weeks, a time course similar to that of EGFP expression. Morphine analgesia is independent of exon 11 variants[9], so this result was prospective. The 6TM variant mMOR-1G restored Exoticin analgesia in the E1/E11 KO mouse, indicating that a $6 \mathrm{TM}$ variant is both necessary and sufficient for its actions. In earlier studies, although the diminished activity of the other drugs in E11 KO mice implicated exon 11 variants in its actions, the inability to rescue their response in the E1/E11 $\mathrm{KO}$ mice indicated that a $6 \mathrm{TM}$ variant was not sufficient, and other factors may be involved. Finally, some drugs may need to express 6TM and 7TM at the same time to be active. For example, the analgesic effect of buprenorphine loses its activity in E11 KO mice[9] and is also significantly reduced in E1/E11 KO mice, which means that the analgesic effect of buprenorphine requires 6TM and 7TM participate.

Opioids are among the most powerful analgesics for treating severe pain, the physicians have to reasonable use opioids to have good management of pain and suffering associated with cancer including management of side effects caused by opioids. Opioids couple opioid receptors and affect several pharmacological effects. In addition to analgesic effects, opioids have side effects such as constipation, nausea and vomiting, and respiratory depression. Mu receptor agonists have been used to control pain and treat side effects caused by opioids, and are even clinically used in an opioid withdrawal syndrome. Our experimental results prove that Exoticin, as a new chemotype for opioid analgesia which does not have opioid-related side effects. 
In summary, the 6TM MOR-1 splice variant mMOR-1G is both necessary and sufficient for Exoticin analgesia. With its broad analgesic activity in thermal, inflammatory, and mechanical pain models[33], as well as its lack of side effect such as respiratory depression, physical dependence, and so on, Exoticin may represent a new class of analgesic different from traditional opioids and $6 \mathrm{TM}$ variants may be an valuable targets or direction for future analgesic drug development.

\section{Author contribution}

F.Q. participated in designing experiments, carried out the experiments in this study, prepared the first draft of this manuscript. Z.L. and Q.W. conceived of the study, participated in its design. A.L., Y.J., Z.L., H.Z., D.N., Q.L., H.W., S.L. performed a part of experiments. Q.L., J.L., S.L. participated in experimental design and consulted on the study. All authors approved the final manuscript.

\section{Conflict of interest}

None of the authors has a conflict of interest related to this research.

\section{Acknowledgments}

We are grateful to Professor YingXian Pan for providing exon $11 \mathrm{mu}$ opioid knockout and exon 1/ exon 11 mu opioid double knockout mice. We also thinks to Professor JingGen Liu (Shanghai institute of Materia Medica, Chinese Academy of Sciences) for providing Binging assays. This work was supported by the grants from the National Natural Science Foundation of China (Grant/Award Number: 81673412) to ZG.L. This study was also funded by the Postgraduate Research \& Practice Innovation Program of Jiangsu Province (KYCX20_1492) to FF.Q.

\section{References}

1. Caudill-Slosberg, M.A., L.M. Schwartz, and S.J.P. Woloshin, Office visits and analgesic prescriptions for musculoskeletal pain in US: 1980 vs. 2000. 2004. 109 (3): p. 514-519.

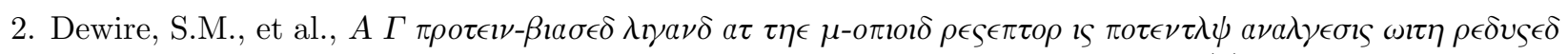

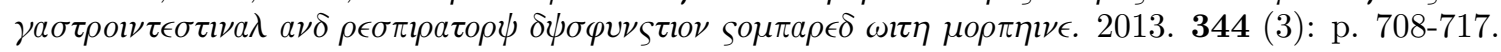

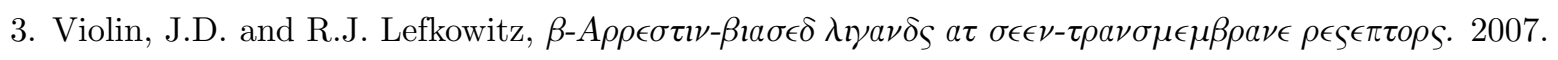

4. Matthes, H.W.D., et al., Loss of morphine-induced analgesia, reward effect and withdrawal symptoms in mice lacking the |[micro]|-opioid-receptor gene. 1996.383 (6603): p. 819.

5. Pasternak, G.W. and Y.X.J.P.R. Pan, Mu Opioids and Their Receptors: Evolution of a Concept. 2013. 65 (4): p. 1257.

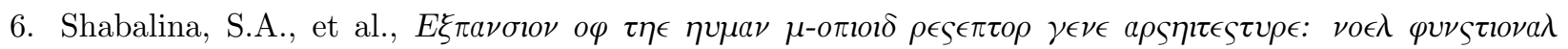

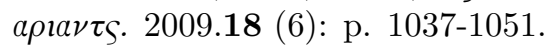

7. Xu, J., et al., Isolating and characterizing three alternatively spliced mu opioid receptor variants: mMOR1A, mMOR-1O, and mMOR-1P. 2014.

8. Xu, J., et al., Identification and characterization of seven new exon 11-associated splice variants of the rat mu opioid receptor gene, OPRM1. 2011.

9. Majumdar, S., et al., Truncated G protein-coupled mu opioid receptor MOR-1 splice variants are targets for highly potent opioid analgesics lacking side effects.

10. Lu, Z., et al., Mediation of opioid analgesia by a truncated 6-transmembrane GPCR. 2015. 125 (7): p. 2626-30.

11. Pan, Y., et al., Involvement of exon 11-associated variants of the mu opioid receptor MOR-1 in heroin, but not morphine, actions.2009. 106 (12): p. 4917-4922. 
12. Olawore, N.O., et al., Chemical composition of the leaf and fruit essential oils of Murraya paniculata (L.) Jack. (Syn. Murraya exotica Linn.). 2005. 20 (1): p. 54-56.

13. Bishay, D., et al., PHYTOCHEMICAL STUDY OF MURRAYA EXOTICA L. CULTIVATED IN EGYPT II- COUMARINS OF THE LEAVES. 1988. 11 (1): p. 88-104.

14. Wu, L., et al., Evaluation of anti-inflammatory and antinociceptive activities of Murraya exotica. 2010. 48 (12): p. 1344-53.

15. F., et al., Composition and antimicrobial activity of the essential oil of Murraya exotica L. 1998.

16. Kong, Y.C., et al., A chemotaxonomic division of Murraya based on the distribution of the alkaloids yuehchukene and girinimbine. 1986.14 (5): p. 491-497.

17. Ganguly, S.N. and A.J.P. Sarkar, Exozoline, a new carbazole alkaloid from the leaves of Murraya exotica. 1978. 17 (10): p. 1816-1817.

18. Teshima, N., et al., Two New Biocoumarins from the Leaves of Murraya exotica. 2005.

19. Shafiullah, S.M., et al., Polymethoxyflavones from Nicotiana plumbaginifolia (Solanaceae) Exert Antinociceptive and Neuropharmacological Effects in Mice. 2018. 9 : p. 85-.

20. Damour, F.E., D.L.J.J.o.P. Smith, and E. Therapeutics, A METHOD FOR DETERMINING LOSS OF PAIN SENSATION. 1941. 72 (1): p. 74-79.

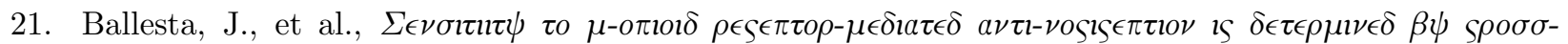

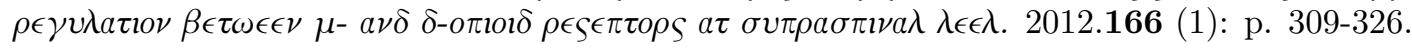

22. Gistrak, M.A., et al., Pharmacological actions of a novel mixed opiate agonist/antagonist: naloxone benzoylhydrazone. 1989.251 (2): p. 469-476.

23. Ling, G.S.F., et al., Separation of morphine analgesia from physical dependence. 1984. 226 (4673): p. 462-464.

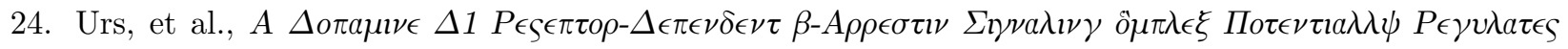

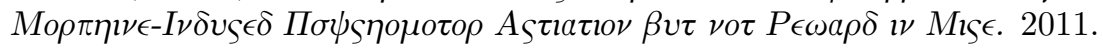

25. Majumdar, S., et al., Generation of novel radiolabeled opiates through site-selective iodination. 2011. 21 (13): p. 4001-4004.

26. Grinnell, S.G., et al., Synthesis and Characterization of Azido Aryl Analogs of IBNtxA for RadioPhotoaffinity Labeling Opioid Receptors in Cell Lines and in Mouse Brain. 2020.

27. Chen, Y., et al., TRPV4 is necessary for trigeminal irritant pain and functions as a cellular formalin receptor. 2014.155 (12): p. 2662-2672.

28. Islam, A., et al., Abuse Liability, Anti-Nociceptive, and Discriminative Stimulus Properties of IBNtxA. 2020. XXXX (XXX).

29. Majumdar, S., et al., Truncated $G$ protein-coupled mu opioid receptor MOR-1 splice variants are targets for highly potent opioid analgesics lacking side effects. 2011. 108 (49).

30. Wise, H.J.J.o.M.S., The roles played by highly truncated splice variants of G protein-coupled receptors. 2012. 7 (1): p. 13-13.

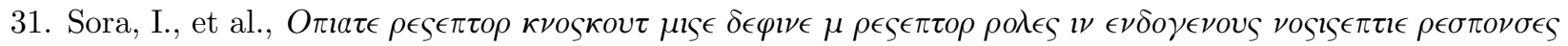

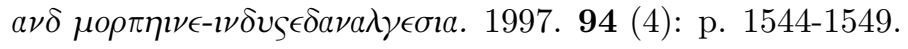

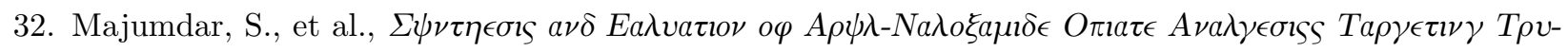

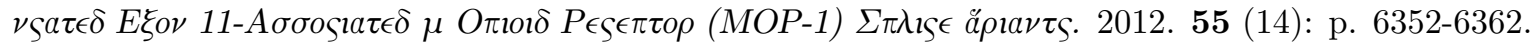


33. Wieskopf, J.S., et al., Broad-spectrum analgesic efficacy of IBNtxA is mediated by exon 11-associated splice variants of the mu-opioid receptor gene. 2014.155 (10): p. 2063-2070.

34. Schuller, A.G.P., et al., Retention of heroin and morphine- 6 beta-glucuronide analgesia in a new line of mice lacking exon 1 of MOR-1. 1999. 2 (2): p. 151.

A

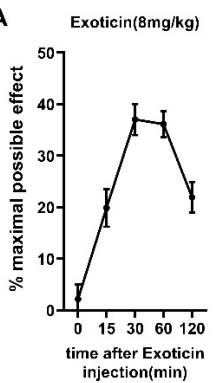

E

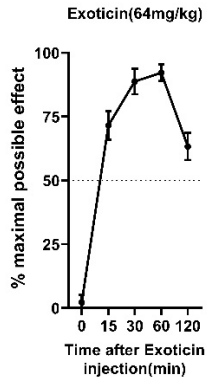

B

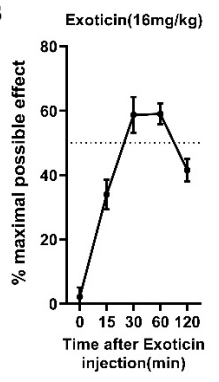

$\mathbf{F}$

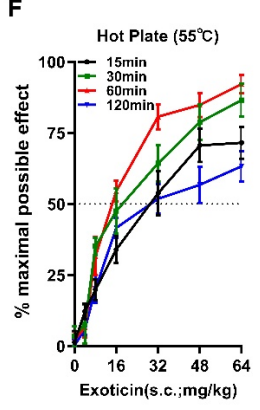

C
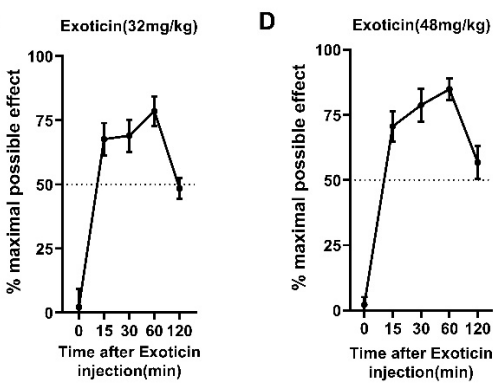

G

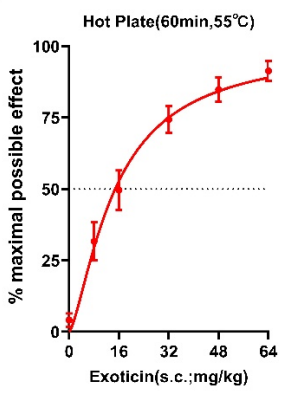

\section{Legend}

Figure 1. Dose-response relationship for the anti-nociceptive effect of s.c. Exoticin evaluated by hot plate in WT mice. (A-F) Time course of hot plate antinociception of different dose Exoticin given subcutaneously in WT mice. Groups of mice were given different doses of Exoticin by subcutaneously and tested for analgesic response at different time points. (G)The cumulative dose-response curves were performed on groups of WT mice for antinociception in the hot plate assay with Exoticin subcutaneously. Animals were tested $15 \mathrm{~min}$ after the Exoticin to generate the analgesic response curve. Each point in graphs represents mean \pm SEM maximum possible effect (\%MPE). Sample sizes are 6 mice per dose, Dotted lines indicate $50 \% \mathrm{MPE}$. 


\section{A. Formalin Test}

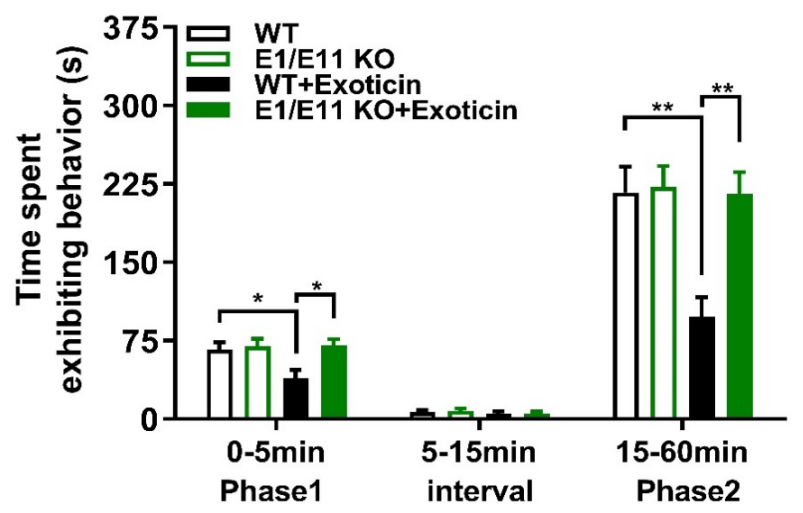

B. CFA

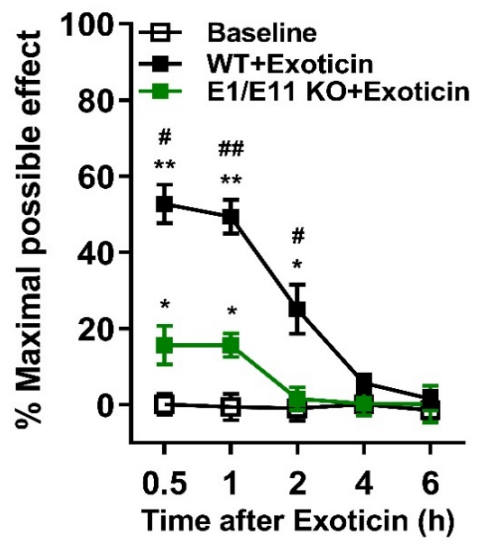

C.

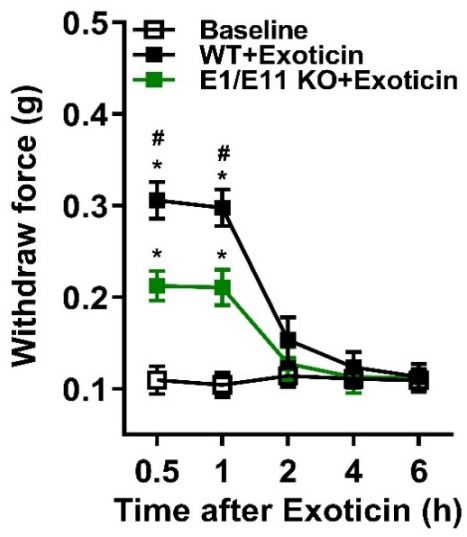

Figure 2. Antinociception of Exoticin in (A) formalin test and (B, C) CFA inflammatory pain test. Mice pre-administrated with Exoticin, spontaneous pain behavior was observed immediately in WT and E1/E11 mice following injection $5 \%$ formalin(A). Symbols in graphs on left represent mean \pm standard error of the mean (SEM) percent samples licking $\left({ }^{*} \mathrm{P}<0.05,{ }^{*} \mathrm{P}<0.05\right)$. Animals received a s.c. injection of Exoticin or vehicle at 4 days after CFA injection in WT and E1/E11 mice, followed by hot plate (B) and mechanical (C) withdrawal assay. Symbols in graphs on left side represent mean \pm SEM maximum possible effect (\%MPE) or withdraw force $\left({ }^{*} \mathrm{P}<0.05\right.$, compared with baseline, ${ }^{\#} \mathrm{P}<0.05$ compared with $\mathrm{E} 1 / \mathrm{E} 11 \mathrm{KO}$ + Exoticin group). Sample sizes are 6 to 8 mice per group. 
A

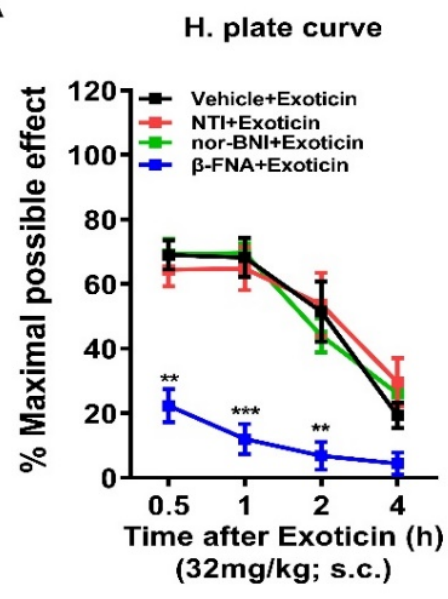

C

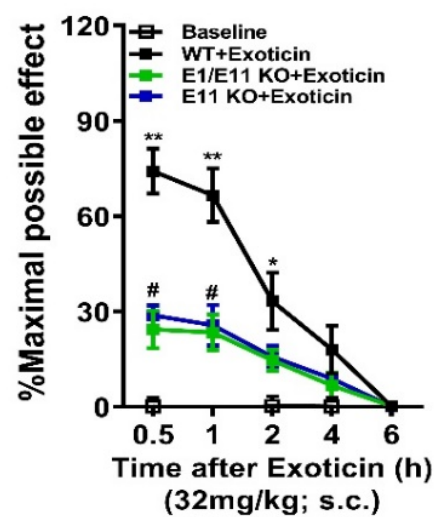

B

\section{Tail-flick}

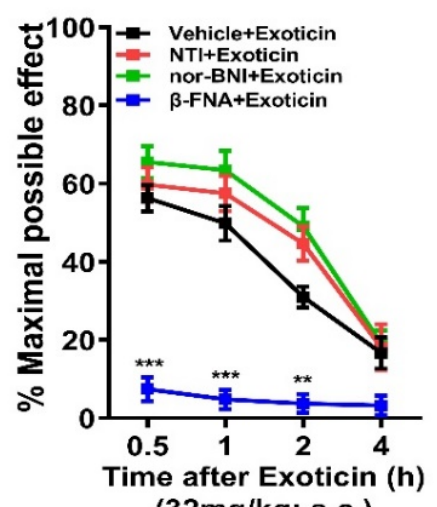

(32mg/kg; s.c.)

D

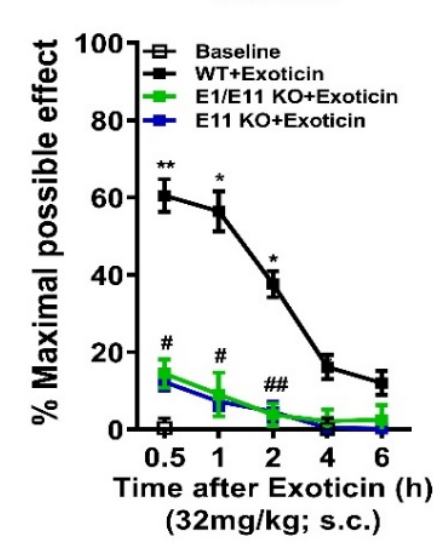

Figure 3. Pharmacological and genetic reversal of antinociception of Exoticin.

Reversal of antinociception by selective antagonists in tai-flick(A) and hot-plate(B). Groups of WT mice $(\mathrm{n}=6)$ received Exoticin $(32 \mathrm{mg} / \mathrm{kg}$ s.c $)$ and the indicated antagonist. $\beta$ - Funaltrexamine $(\beta-F N A ; 40 \mathrm{mg} / \mathrm{kg}$ s. c) and norbinaltorphimine (norBNI; $10 \mathrm{mg} / \mathrm{kg} \mathrm{s}$. c) were administered $24 \mathrm{~h}$ before agonist testing. Naltrindole (NTI; $0.5 \mathrm{mg} / \mathrm{kg} \mathrm{s}$. c), was administered $15 \mathrm{~min}$ before Exoticin. All antinociception testing was performed 15 min after the administration of Exoticin. Exoticin antinociception is insensitive to NTI and nor-BNI, whereas antinociception is antagonized by $\beta$-FNA $\left({ }^{*} \mathrm{P}<0.05\right.$, compared with Vehicle + Exoticin group). Antinociception of Exoticin in WT, E11 KO, and E1/ E11 double KO mice were performed on groups of mice $(\mathrm{n}=5)$ for antinociception in the hot-plate $(\mathrm{C})$ and tail flick assay $(\mathrm{D})$ with Exoticin given subcutaneously. Exoticin played significant antinociceptive effects in WT mice $\left({ }^{*} \mathrm{P}<0.05\right.$, compared with baseline), however, no antinociception was observed in E11 KO and E1/E11 KO mice, suggesting that the antinociceptive effect of Exoticin is mediated by the E11 MOR-1 variants $\left({ }^{\#} \mathrm{P}<0.05\right.$, E11 KO + Exoticin compared with WT + Exoticin group). All values are expressed as the mean $\pm \mathrm{SEM}$. 


\section{A f/BPM}
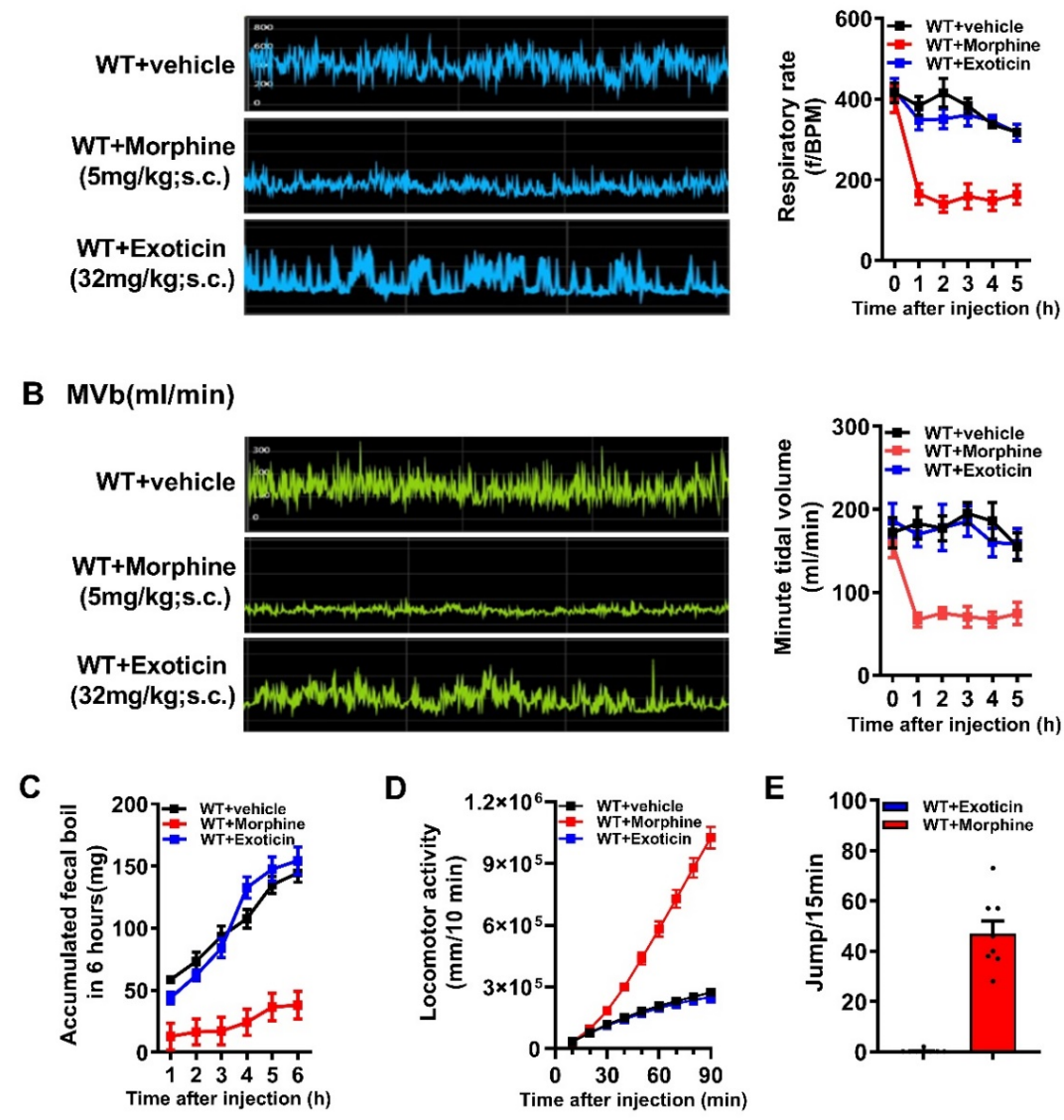

Figure 4. Opioid-related side effects of Exoticin. (A and B). Graph and statistical results of respiratory rate and tidal volume in minutes for each group. Animals were randomly assigned to receive saline $(\mathrm{n}=5)$, Exoticin $(32 \mathrm{mg} / \mathrm{kg}, \mathrm{s}$. c., $\mathrm{n}=5)$ or morphine $(10 \mathrm{mg} / \mathrm{kg}, \mathrm{s}$. c., $\mathrm{n}=5)$. While morphine caused respiratory depression at $5 \mathrm{mg} / \mathrm{kg}$ dose, Exoticin did not depress respiratory rate at $32 \mathrm{mg} / \mathrm{kg}$ dose and was not significantly different from saline. (C). Fecal accumulation. Groups of mice $(\mathrm{n}=8)$ received saline, morphine $(10 \mathrm{mg} / \mathrm{kg})$, or Exoticin $(32 \mathrm{mg} / \mathrm{kg}, \mathrm{s} . \quad$., $\mathrm{n}=8)$ and then individually placed in a Plexiglas box with a wired mesh or grid floor. Fecal were collected in a metal tray and weighed at $1 \mathrm{~h}$ intervals. (D). Locomotor activity. Animals were randomly assigned to receive saline $(\mathrm{n}=10)$, Exoticin $(32 \mathrm{mg} / \mathrm{kg}$, s. c., $\mathrm{n}=14)$ or morphine $(10$ $\mathrm{mg} / \mathrm{kg}$, s. c., $\mathrm{n}=12)$. (E). Physical dependence: Groups of mice were injected daily with Exoticin $(32 \mathrm{mg} / \mathrm{kg}$, s. c., $\mathrm{n}=8)$ or Morphine $(10 \mathrm{mg} / \mathrm{kg} \mathrm{s.} \mathrm{c.,} \mathrm{n}=8)$. Animals were challenged with naloxone $(1 \mathrm{mg} / \mathrm{kg})$ on day 7 of the Exoticin and morphine group. Number of jumps was counted over a $15 \mathrm{~min}$ period post-injection. The response of mice treated with morphine was significantly greater than that of mice treated with Exoticin. 
A

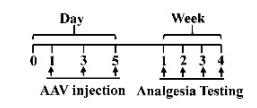

B
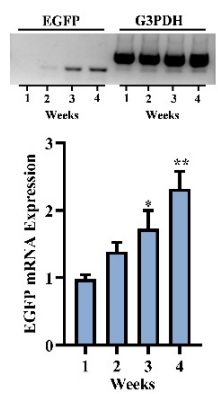

C
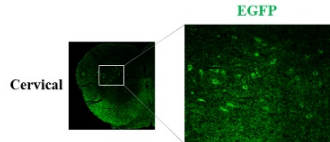

$\beta 3$-tublin
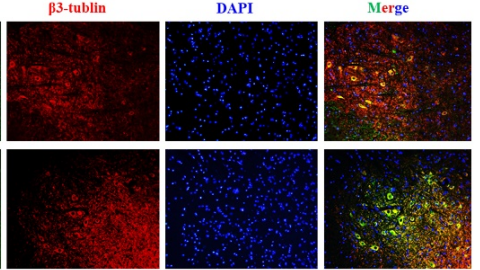

Thoracic
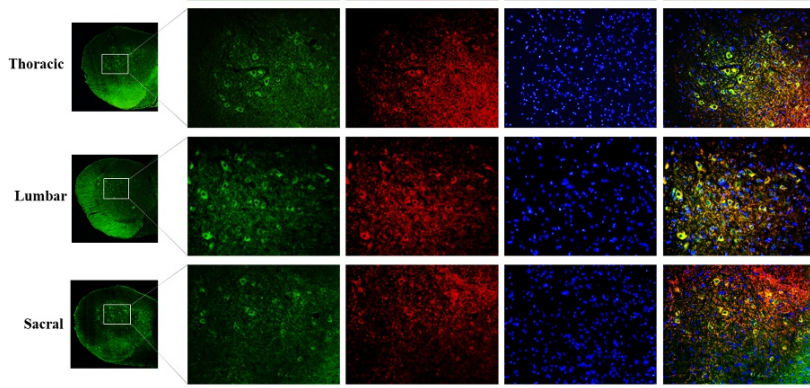

Figure 5. AAV expression in the spinal cord. (A)Schematic of the virus injection paradigm. (B) Time course of spinal EGFP mRNA expression determined by regular PCR (upper panel) and quantified by qPCR (lower panel) $(\mathrm{n}=4) .{ }^{*} \mathrm{p}<0.05,{ }^{* *} \mathrm{p}<0.01$ compared to week 1 . (C) Distribution of AAV EGFP in the spinal cord determined by immunohistochemistry using an anti-GFP antibody. An anti-beta3-Tubulin antibody was used for staining neurons.
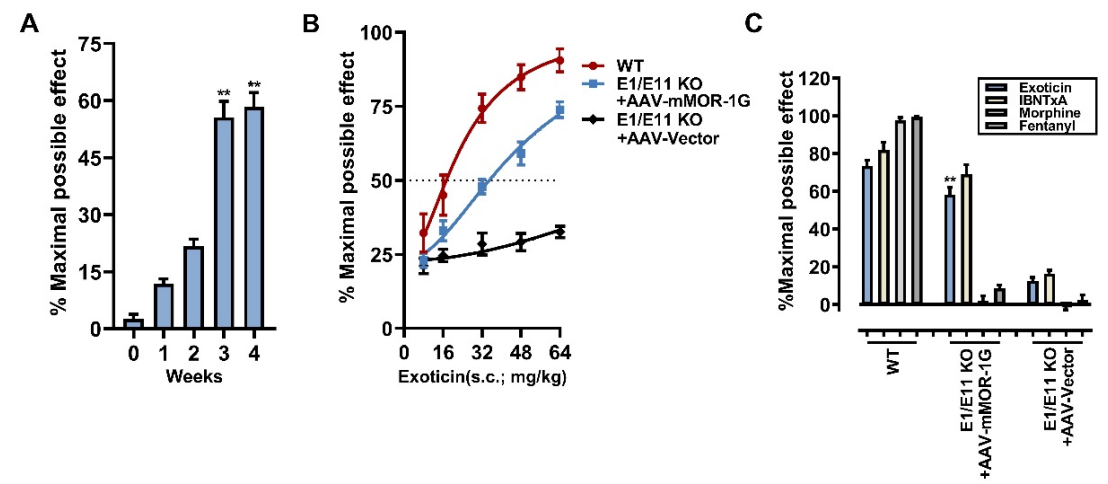

Figure 6. AAV rescue of Exoticin analgesia. (A) Opioid analgesia. Analgesia was determined in groups of mice $(\mathrm{n}=6-8)$ at the stated time. $\left({ }^{* *} \mathrm{P}<0.01\right.$ compared with week 0$)$. (B) Exoticin cumulative dose-response curves were carried out in E1/E11 KO mice with AAV vector alone ( $\mathrm{n}=6)$, AAV-mMOR-1G $(\mathrm{n}=8)$ and WT mice $(\mathrm{n}=8)$.

(C) Single doses of Exoticin (32 mg/kg, s.c., $\mathrm{n}=6)$, morphine $(10 \mathrm{mg} / \mathrm{kg}, \mathrm{s} . \mathrm{c} ., \mathrm{n}=6)$, fentanyl $(0.08 \mathrm{mg} / \mathrm{kg}$, s. c. $\mathrm{n}=6$ ) were administered to groups of either WT or E1/E11 KO mice infected with AAV-mMOR-1G. The mice were assessed for analgesia. ANOVA shows that the Exoticin group and the morphine/fentanyl group in AAV-mMOR-1G animals were significantly different. The Exoticin in WT and AAV-mMOR-1G animals were not significantly different from each other but were different from the E1/E11 KO infected with $\mathrm{AAV}-$ Vector $\left({ }^{* *} \mathrm{P}<0.01\right)$. 


\begin{tabular}{|c|c|c|c|}
\hline Morphine & IBNTxA & Exoticin \\
\hline Charged(negative) \\
Solvent exposure
\end{tabular}

Figure 7. 2D protein-ligand interaction diagram.

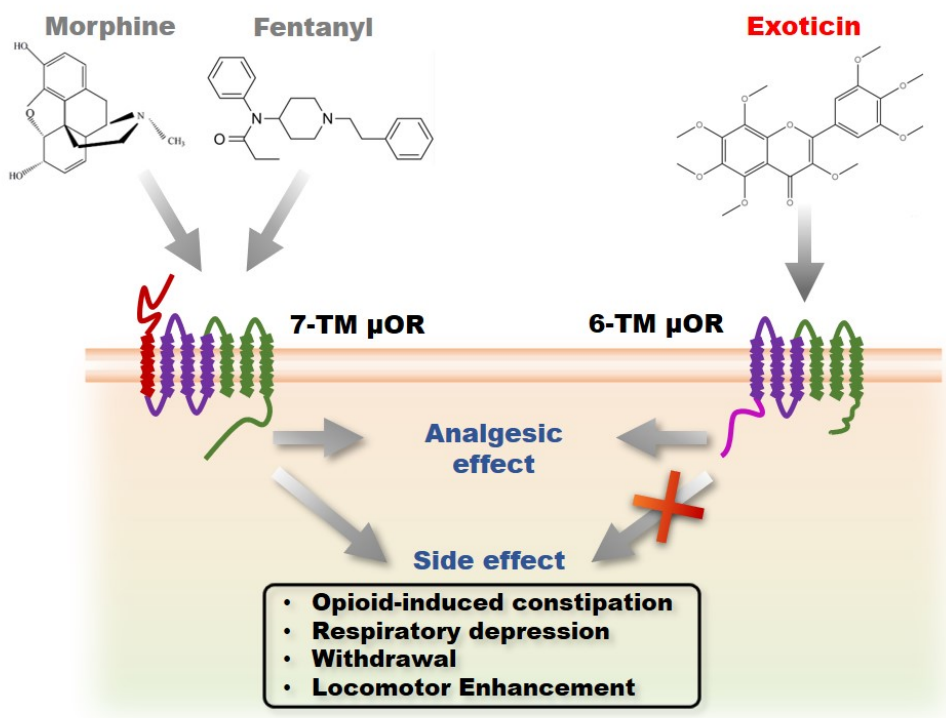

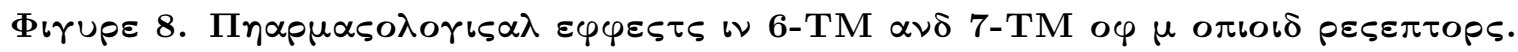

Hosted file

image9.emf available at https://authorea.com/users/371296/articles/489676-mediation-ofexoticin-analgesia-by-a-truncated-6-transmembrane-of-the-mu-opioid-receptor

Table 1. Receptor binding assay for Exoticin. Receptor binding assays were performed using indicated membranes as described in Materials and Method.

\section{Hosted file}

image10.emf available at https://authorea.com/users/371296/articles/489676-mediation-ofexoticin-analgesia-by-a-truncated-6-transmembrane-of-the-mu-opioid-receptor

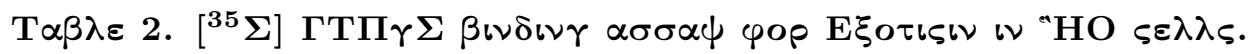

